CERN-PH-TH/2008-231

IFT-UAM/CSIC-08-82

UAB-FT-658

\title{
A Note on Unparticle Decays
}

\author{
Antonio Delgado ${ }^{a}$, José R. Espinosa ${ }^{b, c}$, José Miguel No ${ }^{b}$, Mariano Quirós ${ }^{c, d}$ \\ ${ }^{a}$ Department of Physics, 225 Nieuwland Science Hall, University of Notre Dame, \\ Notre Dame, IN 46556-5670, USA \\ ${ }^{b}$ IFT-UAM/CSIC, Fac. Ciencias UAM, 28049 Madrid, Spain \\ ${ }^{c}$ IFAE, Universitat Autònoma de Barcelona, 08193 Bellaterra, Barcelona, Spain \\ and \\ ICREA, Instituciò Catalana de Recerca i Estudis Avançats, Barcelona, Spain \\ ${ }^{d}$ Theory Division, CERN, Geneva 23 CH-1211, Switzerland
}

\begin{abstract}
The coupling of an unparticle operator $\mathcal{O}_{U}$ to Standard Model particles opens up the possibility of unparticle decays into standard model fields. We study this issue by analyzing the pole structure (and spectral function) of the unparticle propagator, corrected to account for one-loop polarization effects from virtual SM particles. We find that the propagator of a scalar unparticle (of scaling dimension $1 \leq d_{U}<2$ ) with a mass gap $m_{g}$ develops an isolated pole, $m_{p}^{2}-i m_{p} \Gamma_{p}$, with $m_{p}^{2} \lesssim m_{g}^{2}$ below the unparticle continuum that extends above $m_{g}$ (showing that the theory would be unstable without a mass gap). If that pole lies below the threshold for decay into two standard model particles the pole corresponds to a stable unparticle state (and its width $\Gamma_{p}$ is zero). For $m_{p}^{2}$ above threshold the width is non zero and related to the unparticle decay rate into Standard Model particles. This picture is valid for any value of $d_{U}$ in the considered range.
\end{abstract}


Unparticle physics was introduced in Ref. [1] as the effective description of a conformal theory coupled to the Standard Model (SM). Unparticles have their origin in a hidden sector that flows to a strongly coupled conformal theory with an infrared fixed point below some energy scale $\Lambda_{U}$. Since that theory is strongly coupled the anomalous dimensions can be large and (below the scale $\Lambda_{U}$ ) unparticle operators can have a dimension $d_{U}$ which differs sizably from its (integer) ultraviolet dimension. In this note we consider unparticles not charged under the SM gauge group and (in order to enhance its interactions with the Standard Model) with the lowest possible dimension. Therefore we will discuss scalar unparticles $\mathcal{O}_{U}$ with $1 \leq d_{U}<2[2$, , 3].

The conformal invariance of the unparticle sector is explicitly broken by its interactions with the Standard Model. Moreover, when the Higgs field acquires a vacuum expectation value (VEV) this large breaking of conformal invariance gives rise to a mass gap $m_{g}$ in the unparticle spectrum that consists of a continuum of states above $m_{g}$ [4. The mass gap plays a relevant role in the cosmology [5] and phenomenology [6]-[11] of unparticles and it should be taken into account when constraining the unparticle theory from cosmological and experimental data.

In this paper we consider the issue of the stability of unparticles coupled to the Standard Model or, in other words, their possible decay into SM particles. (This is a controversial subject, see [5, 9, 12, 13.) This issue should have a great impact for unparticles in their influence on early Universe cosmology, in their capability as Dark Matter candidates and in their possible detection at high-energy colliders through its production and subsequent decay into SM particles. We will see that the possibility of decay, along with the associated resonant structure, will depend on the precise relationship between the mass gap $m_{g}$ and the SM threshold of the channel to which the unparticle operator is coupled. In particular we will consider the decay of unparticles into SM particles via the Lagrangian coupling $\mathcal{L}=-\kappa_{U} \mathcal{O}_{S M} \mathcal{O}_{U}$ where $\mathcal{O}_{S M}$ is a SM operator which can provide a channel for unparticle decay and $\kappa_{U}$ is a coupling with dimension $4-d_{U}-d_{S M}$. Examples of such SM operators are $F_{\mu \nu}^{2}, m_{f} \bar{f} f$ or $|H|^{2}$.

However, instead of focusing on a particular SM operator, we start by simply considering a toy model with a real scalar $\varphi$, with bare mass $m_{0}$ and zero VEV, coupled to the unparticle scalar operator $\mathcal{O}_{U}$ with scaling dimension $d_{U}$ through the effective Lagrangian

$$
\mathcal{L}_{e f f}=\frac{1}{2}\left(\partial_{\mu} \varphi\right)^{2}-\frac{1}{2} m_{0}^{2} \varphi^{2}-\frac{1}{2} \kappa_{U} \varphi^{2} \mathcal{O}_{U}
$$

which should capture the main features of more realistic channels.

The last term in the Lagrangian above induces a tadpole term for the unparticle operator at one-loop, which would trigger an unparticle VEV 1. This is similar to what happens when the operator $\mathcal{O}_{U}$ is coupled to $|H|^{2}$ and the Higgs field $H$ acquires a VEV (although there the tadpole is a tree level effect). Here we see that this tadpole problem is more generic and would appear even without coupling the unparticles to the Higgs. It was

\footnotetext{
${ }^{1}$ This tadpole is quadratically sensitive to UV physics so that one expects it to be of order $\kappa_{U} \Lambda_{U}^{2} /\left(16 \pi^{2}\right)$.
} 
shown in Ref. [4] that in the presence of such tadpoles an infrared (IR) divergence appears, that has to be cutoff by an IR mass gap $m_{g}$. In the context of [4] the mass gap can be introduced in various different ways such that the conformal invariance is spontaneously broken along with the electroweak symmetry. Here we just assume that such a mass gap is provided by the theory. Of course the VEV of $\mathcal{O}_{U}$ in turn induces a one-loop correction to the mass of the field $\varphi$. We assume that this one-loop corrected mass squared is positive, $m^{2}>0$, so as to keep $\langle\varphi\rangle=0$. An alternative possibility is to impose the renormalization condition of zero unparticle tadpole at one loop so that $\left\langle\mathcal{O}_{U}\right\rangle=0$. As we show later on, a non-zero mass gap will be necessary in any case.

In the presence of the mass gap $m_{g}$ the unparticle propagator reads [1, 2]

$$
-i P_{U}^{(0)}(s)=\frac{1}{D_{U}^{(0)}(s)} \equiv \frac{A_{d_{U}}}{2 \sin \left(\pi d_{U}\right)} \frac{1}{\left(-s+m_{g}^{2}-i \epsilon\right)^{2-d_{U}}},
$$

with

$$
A_{d_{U}}=\frac{16 \pi^{5 / 2}}{(2 \pi)^{2 d_{U}}} \frac{\Gamma\left(d_{U}+1 / 2\right)}{\Gamma\left(d_{U}-1\right) \Gamma\left(2 d_{U}\right)},
$$

where we have explicitly introduced the mass gap $m_{g}$ that breaks the conformal invariance. In fact in some scenarios this parameter can be related to the VEV of the Higgs field, as was shown in Ref. [4]. A spectral function analysis shows that, at this level, the unparticle spectrum is a continuum extending above the mass gap. More precisely, the spectral function, defined as

$$
\rho_{U}^{(0)}(s)=-\frac{1}{\pi} \operatorname{Im}\left[-i P_{U}^{(0)}(s+i \epsilon)\right]
$$

is given by

$$
\rho_{U}^{(0)}(s)=\frac{A_{d_{U}}}{2 \pi}\left(s-m_{g}^{2}\right)^{d_{U}-2} \theta\left(s-m_{g}^{2}\right) .
$$

The polarization $\Sigma(s)$ induced in the unparticle propagator by the one-loop diagram exchanging $\varphi$-fields can be simply added by a Dyson resummation to give

$$
-i P_{U}^{(1)}=\frac{1}{D_{U}^{(1)}(s)}=\frac{1}{D_{U}^{(0)}(s)+\Sigma(s)} .
$$

The polarization $\Sigma(s)$ is given in the $\overline{M S}$-renormalization scheme by [14]

$$
\Sigma(s)=\frac{\kappa_{U}^{2}}{32 \pi^{2}}\left\{\log \left(\frac{\Lambda_{U}^{2}}{m^{2}}\right)+2-2 \lambda(s) \log \left[\frac{1+\lambda(s)}{\sqrt{\lambda^{2}(s)-1}}\right]\right\},
$$

where $\lambda(s)=\sqrt{1-4 m^{2} / s}$ and we have set the renormalization scale equal to the cutoff $\Lambda_{U}$. (For numerical work we fix $\Lambda_{U}=100 \mathrm{~m}$ ).

The location of the unparticle resonances will be determined by the propagator poles $s=m_{p}^{2}-i m_{p} \Gamma_{p}$ in the complex $s$-plane (with $m_{p}$ the pole mass and $\Gamma_{p}$ its width). The polarization $\Sigma(s)$ has a branch cut that we take from the threshold at $s=4 m^{2}$ to infinity along the real axis with the principal Riemann sheet corresponding to $0 \leq \theta \leq 2 \pi$, where 
$\theta$ is defined as $s-4 m^{2}=\left|s-4 m^{2}\right| e^{i \theta}$. The second Riemann sheet is reached by shifting $\theta \rightarrow \theta+2 \pi$. It can be easily seen that a change in the Riemann sheet is equivalent to the replacement $\lambda(s) \rightarrow-\lambda(s)$. Then since the complete propagator is a function of $\lambda$

$$
D^{(1)}(s) \equiv \mathcal{D}[s, \lambda(s)]
$$

the pole equations

$$
\mathcal{D}\left[s, \epsilon_{R} \lambda(s)\right]=0
$$

where $\epsilon_{R}=1(-1)$ correspond to solutions in the first (second) Riemann sheet [15].

A numerical analysis of the pole equation (9) shows that, besides the unparticle continuum, an isolated pole appears. Note that the tree-level propagator had no pole $\left(m_{g}^{2}\right.$ is not a pole but a branch point) and therefore the pole appearance is a purely one-loop effect. Due to the sign of this radiative effect we find that $m_{p}^{2}$ is always 2 below $m_{g}^{2}$, but quite close to it as the polarization is a radiative effect: $m_{p}^{2} \lesssim m_{g}^{2}$. For $m_{p} \leq 2 m$ this isolated pole is real $\left(\Gamma_{p}=0\right)$ and located in the first Riemann sheet. Such pole does not correspond to any decaying unparticle and it is entirely due to the fact that $\Sigma \neq 0$ below the threshold and could be interpreted as an unparticle bound state. We show in Fig. 1 [left panel] a plot of $m_{p}$ vs. $d_{U}$ for $m=m_{g}$. In this plot one can see that indeed $m_{p} \rightarrow m_{g}$ for $d_{U} \rightarrow 2$.
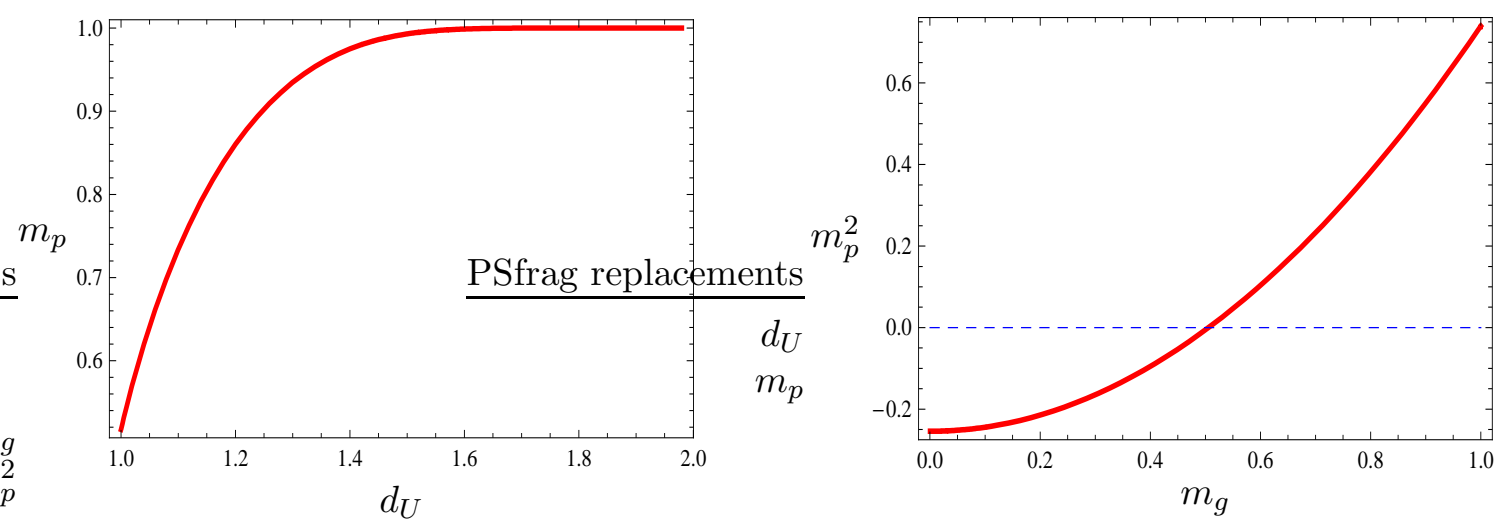

Figure 1: Left panel: Plot of $m_{p}$ as a function of $d_{U}$ for $\kappa_{U}=5, m_{g}=m$ and $\mu=\Lambda_{U}=100 \mathrm{~m}$. Right panel: Plot of $m_{p}^{2}$ vs. $m_{g}$ for $\kappa_{U}=5$ and $d_{U}=1.2$. All masses are in units of $m$.

An immediate consequence of the negative mass shift responsible for $m_{p}^{2}<m_{g}^{2}$ is that it yields a lower bound on the scale of conformal breaking $m_{g}$. That bound is related to the masses of the Standard Model particles the unparticle operator is coupled to ( $m$ in our case). This fact is shown by Fig. 1 [right panel], where the pole squared mass $m_{p}^{2}$ is plotted vs. $m_{g}$ for $d_{U}=1.2$. We can see that the isolated unparticle pole becomes tachyonic for

\footnotetext{
${ }^{2}$ For $d_{U}$ very close to 2 one can also have $m_{p}>m_{g}$, but in such cases the mass difference between the pole and the mass gap is infinitesimal.
} 
small values of $m_{g}\left(m_{g}<0.5 \mathrm{~m}\right)$. Moreover, this shows that in the particular limit $m_{g} \rightarrow 0$ the theory becomes unstable. Later on we give an analytical formula for this lower bound on the mass gap.

For $m_{g}>2 m$ the isolated unparticle pole is complex $\left(\Gamma_{p}>0\right)$ and appears in the second Riemann sheet 3 , and this now corresponds to the decay of a resonance. This case is exhibited in Fig. 2, where $m_{p}$ and $\Gamma_{p}$ are plotted vs. $d_{U}$ for the case $m_{g}=4 m$ (thick solid lines). Finally, since $\kappa_{U}^{2}$ is a global factor in the polarization, the values of $m_{g}^{2}-m_{p}^{2}$ and $\Gamma_{p}$ exhibit an approximate scaling behaviour with $\kappa_{U}^{2}$.
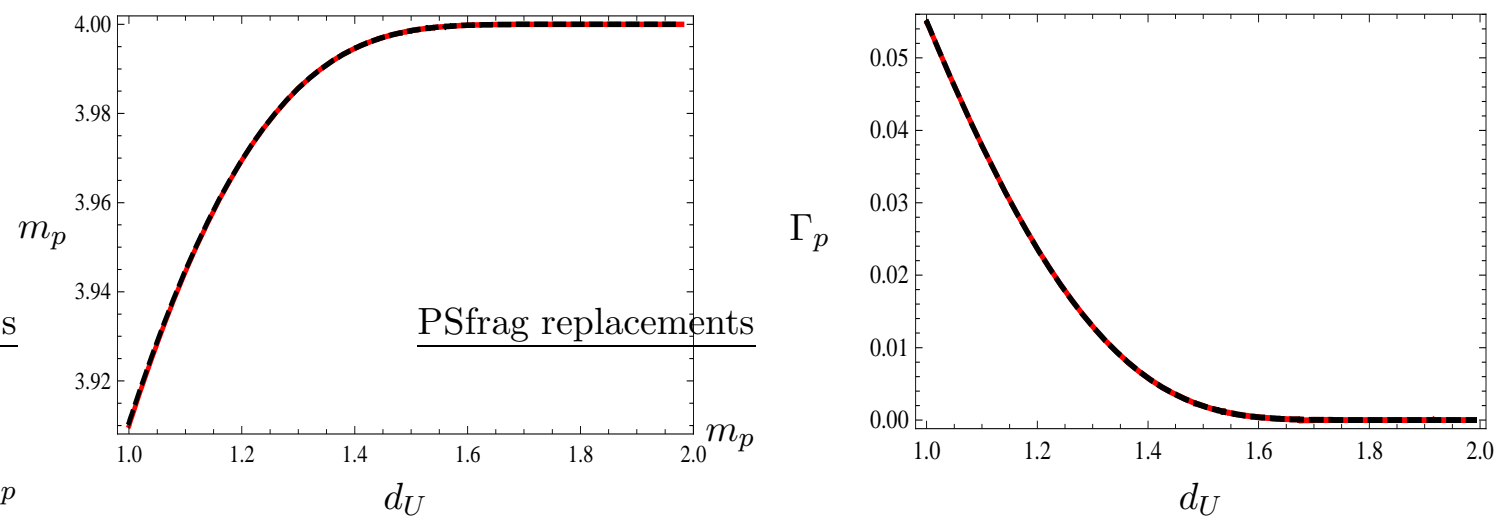

Figure 2: Left [right] panel: Plot of $m_{p}\left[\Gamma_{p}\right]$ as a function of $d_{U}$ for $\kappa_{U}=5$ and $m_{g}=4 m$ (thick solid). Corresponding results based on the analytical approximation of Eq. (11) are plotted in thick dashed lines. All masses are in units of $m$.

We want to emphasize here that there are complex pole solutions for all values of $d_{U}$ in the considered range $1 \leq d_{U}<2$, unlike what was claimed in Ref. [13. In our case, nothing special happens for $d_{U}>3 / 2$ and $m_{p}^{2}$ and $\Gamma_{p}$ smoothly approach $m_{g}^{2}$ and zero respectively when $d_{U} \rightarrow 2$.

It is easy to understand analytically our results. For values of $s$ close to the resonance region one can approximate the complex polarization by the constant

$$
\Sigma(s) \simeq \Sigma\left(m_{g}^{2}\right)=\frac{\kappa_{U}^{2}}{32 \pi^{2}}\left\{\log \left(\frac{\Lambda_{U}^{2}}{m^{2}}\right)+2-\lambda\left(m_{g}^{2}\right)\left[\log \frac{1+\lambda\left(m_{g}^{2}\right)}{1-\lambda\left(m_{g}^{2}\right)}-i \pi\right]\right\},
$$

and a simple calculation yields an analytic approximation for the pole mass and width as

$$
\begin{aligned}
m_{p}^{2} & \simeq m_{g}^{2}-\delta m^{2} \cos \alpha, \\
m_{p} \Gamma_{p} & \simeq \lambda\left(m_{g}^{2}\right) \delta m^{2}|\sin \alpha|
\end{aligned}
$$

where

$$
\delta m^{2} \equiv\left[\frac{\left|\Sigma\left(m_{g}^{2}\right)\right| A_{d_{U}}}{2\left|\sin \left(\pi d_{U}\right)\right|}\right]^{\frac{1}{2-d_{U}}}
$$

\footnotetext{
${ }^{3}$ In all cases we also found the corresponding shadow pole [16] in the unphysical sheet as required by hermitian analyticity.
} 
and

$$
\alpha=\frac{1}{\left(2-d_{U}\right)} \arctan \frac{\operatorname{Im}\left[\Sigma\left(m_{g}^{2}\right)\right]}{\operatorname{Re}\left[\Sigma\left(m_{g}^{2}\right)\right]} .
$$

Figure 2 compares the values for $m_{p}$ and $\Gamma_{p}$ obtained using the analytic approximation in (11) (dashed thick lines) with the full numerical results (thick solid lines) showing that the analytical approximation is excellent. We can use this approximation to write down analytically the lower bound on $m_{g}^{2}$ to avoid a tachyon. It is given by

$$
m_{g}^{2}>\left[\frac{\Sigma(0) A_{d_{U}}}{2\left|\sin \left(\pi d_{U}\right)\right|}\right]^{\frac{1}{2-d_{U}}}
$$

with $\Sigma(0)=\kappa_{U}^{2} /\left(16 \pi^{2}\right) \log \left(\Lambda_{U} / m\right)$.

We can gain further insight on the unparticle spectrum by calculating the spectral function for the one-loop corrected propagator

$$
\rho_{U}(s)=-\frac{1}{\pi} \operatorname{Im}\left[-i P^{(1)}(s+i \epsilon)\right] .
$$

As we show below, this spectral function will reproduce faithfully the main features of the pole structure discussed previously, giving also information on the unparticle continuum above the mass gap. The expression we find for this spectral function is the following:

$$
\rho_{U}(s)=\frac{1}{\pi} \frac{\operatorname{Im}[\Sigma(s)]}{\left|D_{U}^{(1)}(s)\right|^{2}}+\theta\left(4 m^{2}-m_{p}^{2}\right) \frac{\delta\left(s-m_{p}^{2}\right)}{d D_{U}^{(1)}(s) / d s}+\theta\left(s-m_{g}^{2}\right) \frac{2 \sin ^{2}\left(\pi d_{U}\right)}{\pi A_{d_{U}}} \frac{\left(s-m_{g}^{2}\right)^{2-d_{U}}}{\left|D_{U}^{(1)}(s)\right|^{2}} .
$$

The first term of $\rho_{U}(s)$ is proportional to the imaginary part of $\Sigma(s)$ [which contains a factor $\theta\left(s-4 m^{2}\right)$ ] and thus for $m_{p}^{2}>4 m^{2}$ it corresponds, through the Cutkosky rules, to a width for the unparticles which decay beyond the threshold. The second term (for $m_{p}^{2}<4 m^{2}$ ) corresponds to a real pole in the first Riemann sheet, and should be interpreted as a stable (un)particle of mass $m_{p}$. Finally, the third term is proportional to the imaginary part of $D_{U}^{(0)}(s)$ 4 and does not correspond to any unparticle decay, but gives rise to the familiar continuous contribution to the spectral function above the mass gap (a similar continuum appears in the Higgs spectral function when the Higgs is coupled to an unparticle operator [17]).

When the decay $\mathcal{O}_{U} \rightarrow \varphi \varphi$ occurs it should give rise to a resonant structure in the spectral function $\rho_{U}(s)$ through the term proportional to $\operatorname{Im}[\Sigma(s)]$, with an approximate Breit-Wigner distribution centered around $m_{p}^{2}$ of width $\Gamma_{p}$. This should be in correspondence with the structure of the poles of the propagator $P^{(1)}(s)$ in the complex $s$-plane, i.e. to the zeroes of the function $D^{(1)}(s)$ which we have previously studied.

In the left panel of Fig. 3 we have plotted the spectral function for the case of Fig. 1 in which there is no resonant interpretation, but instead a real pole appears. We see a delta

\footnotetext{
${ }^{4}$ Notice that this imaginary part is only different from zero for $s>m_{g}^{2}$ and thus it contains a factor $\theta\left(s-m_{g}^{2}\right)$.
} 
function corresponding to that pole and a continuous component for $s>m_{g}^{2}$. In the right panel of Fig. 3 we have plotted the strength of the isolated pole, $K^{2}\left(m_{p}^{2}, d_{U}\right)$, defined as

$$
K^{2}\left(s, d_{U}\right)=\frac{1}{d D_{U}^{(1)}(s) / d s}
$$
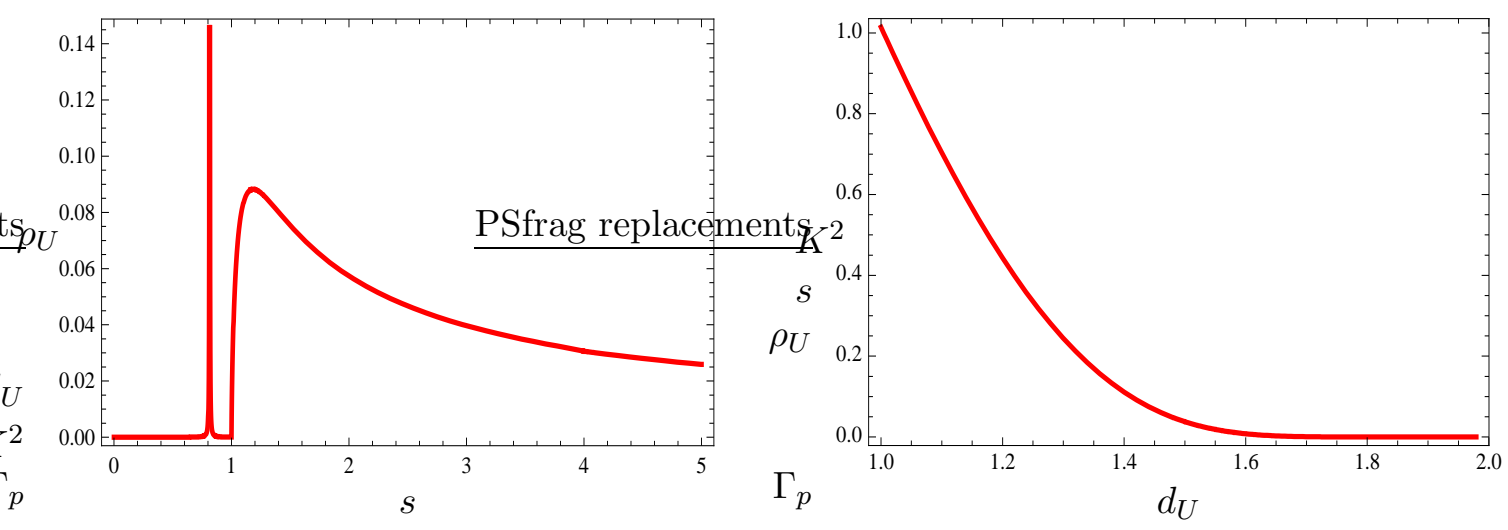

Figure 3: Left panel: Plot of $\rho_{U}(s)$ for $m_{g}=m, d_{U}=1.25, \kappa_{U}=5$ and $\mu=\Lambda_{U}=100 m$. Right panel: Plot of $K^{2}\left(d_{U}\right)$ for the same values of mass parameters. All masses are in units of $m$.

The fact that for $m_{g}>2 m$ the pole width is sharpening for increasing values of $d_{U}$ (as shown by the right plot in Fig. 2) is also shown in Fig. 4, in which we plot the spectral function for values of the dimension $d_{U}=1.25$ [left panel] and $d_{U}=1.5$ [right panel]. In both cases we see a clear resonant contribution that overwhelms the continuous one. In this region and for values of $s$ close to the value of $m_{p}^{2}$ the unparticle behaves as a resonance

It can be easily calculated that the height of the peak is independent of $d_{U} 5$ and given by the simple expression:

$$
\rho_{U}^{\max } \simeq \frac{32}{\kappa_{U}^{2} \lambda\left(m_{g}^{2}\right)}
$$

Therefore, as the width goes to zero we do not recover a Dirac delta function at $m_{p}$ and the resonance will be very difficult to detect experimentally over the continuous background starting at $m_{g}$.

Notice that for $m_{g}^{2}>4 m^{2}$ the resonant ("on-shell") production of unparticles would dominate the amplitude $\varphi \varphi \rightarrow \varphi \varphi$ as it happens with ordinary exchange of particles in the s-channel. Here the presence of unparticles should be detected through a peak in the invariant mass distribution of the final state similar to the case of a new particle resonance (e.g. the production of a $Z^{\prime}$ ). For the case $m_{p}^{2}<4 m^{2}$ the resonance is located below the production threshold and the spectral function is dominated by the continuous contribution, which does not provide any decay. In that case there is no resonant production

\footnotetext{
${ }^{5}$ This statement is true up to values of $d_{U}$ very close to 2 , for which the width of the resonance is zero and $m_{p}=m_{g}$ for all practical purposes.
} 

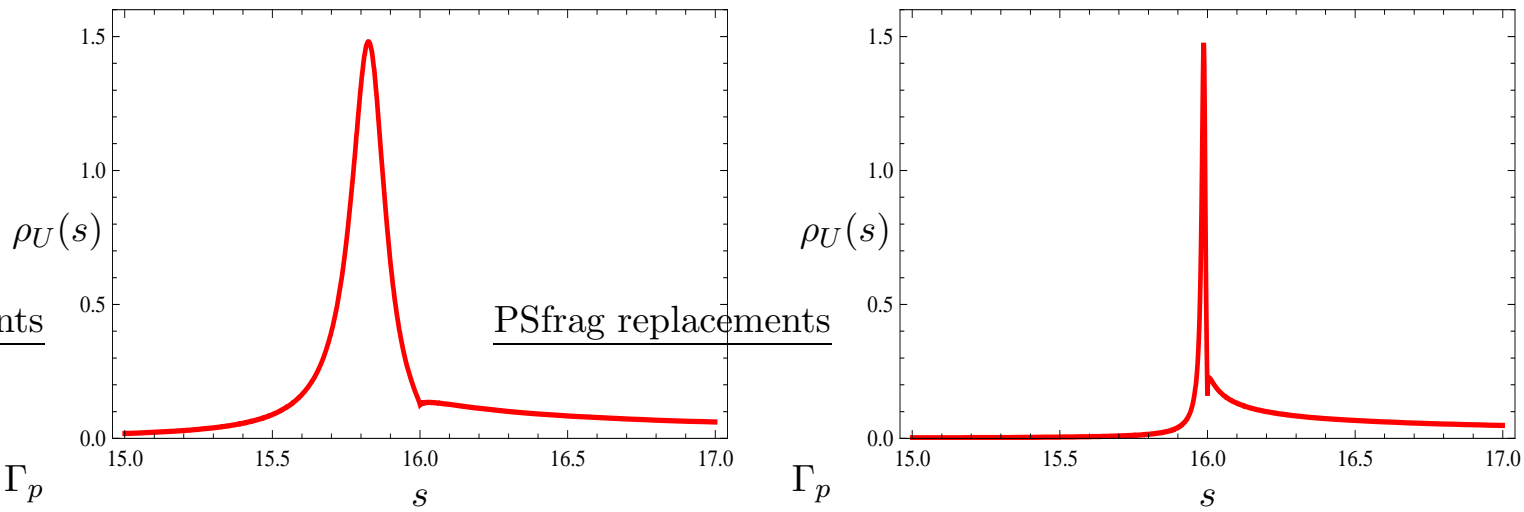

Figure 4: Left [right] panel: Plot of $\rho_{U}(s)$ for $d_{U}=1.25\left[d_{U}=1.5\right], m_{g}=4 m, \kappa_{U}=5$ and $\mu=\Lambda_{U}=100 \mathrm{~m}$. All masses are in units of $m$.

and the production of the final state $\varphi \varphi$ will be as if induced "off-shell". The presence of unparticles in the intermediate state should be detected by the continuous enhancement of the corresponding cross-section. This situation is reminiscent of the familiar case of exchange of graviton Kaluza-Klein modes in ADD theories of extra dimensions where the excess of cross section is used to put bounds on the value of the fundamental scale.

The formalism to be used for any realistic Standard Model channel as e.g. $A_{\mu} A_{\nu}$, $\bar{\psi}_{L} \psi_{R}$ or $H^{\dagger} H$, is similar to the one used in the toy model considered in this paper. In every case, for the particular channel $\mathcal{O}_{U} \rightarrow A B$, if $m_{p}^{2}>\left(m_{A}+m_{B}\right)^{2}$ the unparticle should be detected in the corresponding cross-section through a peak in the invariant mass distribution of the final state which should reconstruct the resonant pole, much like the reconstruction of a $Z^{\prime}$ resonance. On the contrary if $m_{p}^{2}<\left(m_{A}+m_{B}\right)^{2}$ then the only indirect detection of the unparticle should be by an excess of events with respect to the corresponding Standard Model cross-section.

In the Standard Model the only relevant (unsuppressed) operator which can give rise to unparticle two-body decays is $\kappa_{U}|H|^{2} \mathcal{O}_{U}$, an interaction which has been thoroughly analyzed in Refs. [4, 17]. In that case the mixing in the broken phase provided by the Lagrangian term $\kappa_{U} v h \mathcal{O}_{U}$ gives rise to a tree-level mixing between the Higgs and unparticles which is $\mathcal{O}\left(\kappa_{U}^{2} v^{2}\right)$. Since this mixing is of the same order as the one loop polarization, $\mathcal{O}\left(\kappa_{U}^{2}\right)$, it can be resummed in the unparticle propagator along with $\Sigma$ leading to [4]

$$
\mathcal{D}_{U}[s, p(s)] \rightarrow \mathcal{D}_{U}[s, p(s)]+\kappa_{U}^{2} \frac{v^{2}}{s-m_{h}^{2}+i \epsilon},
$$

where $m_{h}$ is the tree-level (unmixed) Higgs mass. The analysis should follow similar lines to those presented in this paper (after including the extra mixing term) by just replacing $m \rightarrow m_{h}$. We have checked that the qualitative results found in this paper do not change after the inclusion of the Higgs-unparticle mixing. Finally for other channels corresponding to Standard Model particles which do not acquire any vacuum expectation values the qualitative results should be similar to those presented in this paper. 
To summarize our results, we have studied unparticle decays into SM particles, and showed that this possibility is controlled by the relation between the unparticle mass gap $m_{g}$ and the production threshold $m_{A}+m_{B}$ (the latter are the masses of the decay products). When $m_{g}>m_{A}+m_{B}$ there is enough phase space, unparticles can decay into SM particles, and that decay is accounted for by the appearance of a complex pole on the unparticle one-loop resummed propagator. If in turn $m_{g}<m_{A}+m_{B}$ there is still a pole but with no imaginary part, corresponding to a stable unparticle. Finally, it should be stressed that the pole is always below $m_{g}$ implying that a theory without mass gap and coupled to a SM channel shows an instability. This can be interpreted as a signal that a mass gap should be present once the unparticle is coupled to SM fields.

\section{Appendix A. Normalization of the Spectral Function}

In this appendix we address the issue of the normalization of the spectral function $\rho_{U}(s)$. The integral of a spectral function along the real axis is determined by the normalization of the state being considered. For instance, if we take the tree level spectral function $\rho_{U}^{(0)}$ we would write

$$
N_{U}^{(0)}=\int_{0}^{\infty} \rho_{U}^{(0)}(s) d s=\langle U \mid U\rangle
$$

where $|U\rangle$ represents the unparticle operator $\mathcal{O}_{U}$. This integral turns out to be UV divergent as corresponds to a non-normalizable state $|U\rangle$. Strictly speaking one should cut-off this integral at a scale of order $\Lambda_{U}$, beyond which the theory leaves the conformal regime. The normalization integral scales as

$$
N_{U}^{(0)}\left(\Lambda_{U}\right)=\int_{0}^{\Lambda_{U}^{2}} \rho_{U}^{(0)}(s) d s \sim\left(\Lambda_{U}^{2}\right)^{d_{U}-1}
$$

By using the Cauchy theorem (and the absence of complex poles in the principal Riemann sheet) one can see that $N_{U}^{(0)}\left(\Lambda_{U}\right)$ is proportional to the integral of the propagator $P_{U}\left(p^{2}\right)$ along a circle of radius $\Lambda_{U}^{2}$ so that its scaling is directly dependent on the UV behaviour of such propagator.

After including one-loop polarization effects the shape of the spectral function is affected but it keeps the same normalization as before. In fact, defining

$$
N_{U}\left(\Lambda_{U}\right)=\int_{0}^{\Lambda_{U}^{2}} \rho_{U}(s) d s
$$

one finds

$$
\frac{N_{U}}{N_{U}^{(0)}}=1+\mathcal{O}\left(\frac{m_{g}^{2}}{\Lambda_{U}^{2}}\right)+\mathcal{O}\left(\frac{\kappa_{U}^{2}}{\left(\Lambda_{U}^{2}\right)^{2-d_{U}}} \log \Lambda_{U}^{2}\right),
$$

which tends to 1 for $\Lambda_{U}^{2} \gg m_{g}^{2},\left(\kappa_{U}^{2}\right)^{1 /\left(2-d_{U}\right)}$.

One can also show that

$$
N_{U}\left(\Lambda_{u}\right)-N_{U}^{(0)}\left(\Lambda_{U}\right)=\int_{0}^{\Lambda_{U}^{2}}\left[\rho_{U}(s)-\rho_{U}^{(0)}(s)\right] d s \sim\left(\Lambda_{U}^{2}\right)^{2 d_{U}-3}
$$


which tends to 0 for $d_{U}<3 / 2$, case in which the equality of the normalizations holds also in this stronger sense.

\section{Acknowledgments}

We are indebted to Rafel Escribano for useful discussions about the pole structure of the propagator. J.M.N. thanks the Department of Physics of the University of Notre Dame for hospitality during the last stage of this work. Work supported in part by the European Commission under the European Union through the Marie Curie Research and Training Networks "Quest for Unification" (MRTN-CT-2004-503369) and "UniverseNet" (MRTNCT-2006-035863); by the Spanish Consolider-Ingenio 2010 Programme CPAN (CSD200700042); by a Comunidad de Madrid project (P-ESP-00346); and by CICYT, Spain, under contracts FPA 2007-60252 and FPA 2008-01430.

\section{References}

[1] H. Georgi, Phys. Rev. Lett. 98 (2007) 221601 hep-ph/0703260]; Phys. Lett. B 650, 275 (2007) [hep-ph/0704.2457].

[2] P. J. Fox, A. Rajaraman and Y. Shirman, Phys. Rev. D 76, 075004 (2007) [hep$\mathrm{ph} / 0705.3092]$.

[3] B. Grinstein, K. A. Intriligator and I. Z. Rothstein, Phys. Lett. B 662, 367 (2008) [hep-ph/0801.1140].

[4] A. Delgado, J. R. Espinosa and M. Quirós, JHEP 0710, 094 (2007) [hep$\mathrm{ph} / 0707.4309]$.

[5] J. McDonald, [hep-ph/0709.2350]; [hep-ph/0805.1888].

[6] T. G. Rizzo, JHEP 0710, 044 (2007) [hep-ph/0706.3025]; [hep-ph/0809.4659].

[7] M. Bander, J. L. Feng, A. Rajaraman and Y. Shirman, Phys. Rev. D 76, 115002 (2007) [hep-ph/0706.2677].

[8] V. Barger, Y. Gao, W. Y. Keung, D. Marfatia and V. N. Senoguz, Phys. Lett. B 661, 276 (2008) [hep-ph/0801.3771].

[9] M. J. Strassler, [hep-ph/0801.0629].

[10] G. Cacciapaglia, G. Marandella and J. Terning, JHEP 0801, 070 (2008) [hepph/0708.0005]; [hep-ph/0804.0424]; D. Stancato and J. Terning, [hep-ph/0807.3961].

[11] A. Rajaraman, [hep-ph/0809.5092].

[12] M. A. Stephanov, Phys. Rev. D 76, 035008 (2007) [hep-ph/0705.3049]; 
[13] A. Rajaraman, [hep-ph/0806.1533].

[14] B. A. Kniehl, Phys. Rept. 240, 211 (1994).

[15] R. Escribano, A. Gallegos, J. L. Lucio M, G. Moreno and J. Pestieau, Eur. Phys. J. C 28, 107 (2003) hep-ph/0204338.

[16] P.V. Landshoff, Nuovo Cimento 28, 123 (1963).

[17] A. Delgado, J. R. Espinosa, J. M. No and M. Quirós, JHEP 0804, 028 (2008) [hepph/0802.2680]; [hep-ph/0804.4574]. 